\title{
Telemedicine solutions, a window of opportunity to bridge the gap in maternal and neonatal health services
}

\begin{abstract}
A narrative review was conducted to examine the increased use of telemedicine solutions during COVID-19 pandemic and to discuss the benefits of using telemedicine techniques in the reduction of maternal and neonatal mortalities. Telemedicine has evolved significantly in the past couple of years since the outbreak of COVID-19. One of the areas where telemedicine can save lives is though remote connection with a higher-level facility or more skilled service providers that can deal with emergency obstetric care.

Telemedicine use in emergency obstetric care can significantly reduce maternal and neonatal mortalities and morbidities in remote and underserved areas, and in facilities that have poor trained service providers.

The advantages of applying telemedicine solutions in complicated child births are to overcome long distances, geographic isolation, lack of services, and lack of skilled personnel. It's cost-effective, and more important saves lives. Disadvantages are mainly related to technological infrastructure, availability of an Internet, hardware, financial incentives to on-call recipients of the cases, and their commitment.

The main functionalities of the telemedicine solution to respond to emergency and complicated childbirth cases are proposed to include video conference between health professionals in underserved and specialized service delivery points, sensor's data for electrocardiogram, cardiotocography, ultrasound, and patient data recording. It needs to come with a mobile application to be used by specialists to enable them respond to and manage the emergencies.
\end{abstract}

Keywords: maternal health, neonatal health, telemedicine
Volume 8 Issue I - 2022

\author{
Maha Abdel Wanis \\ United Nations Population Fund - UNFPA, Egypt
}

Correspondence: Maha Abdel Wanis, United Nations Population Fund - UNFPA, Cairo, Egypt, Email abdelwanis@unfpa.org

Received: January 20, 2022 | Published: February 15, 2022

\section{Introduction}

Telemedicine is a service that seeks to improve a patient's health by permitting two-way, real-time interactive communication between the patient and the physician at a distant site. ${ }^{1}$

As stated by WHO, "the delivery of health care services, where distance is a critical factor, by all health care professionals using information and communication technologies for the exchange of valid information for diagnosis, treatment and prevention of disease and injuries, research and evaluation, and for the continuing education of health care providers, all in the interests of advancing the health of individuals and their communities". ${ }^{2}$ The use of telemedicine solutions, can be wither between health professionals to widen their medical knowledge, and can be between health professionals and patients. $^{3}$

Since the outbreak of COVID-19 there was a remarkable increased use of telemedicine solutions, and specifically for maternal and neonatal care. ${ }^{4}$ This approach can fill gaps in delivering and seeking maternal and child health care services, and accordingly reduce maternal mortalities, as it will ensure timely delivery of life-saving services.

In low and middle income countries, particularly in underserved areas, telemedicine solutions are mainly used to connect health service providers with specialists, more advanced referral hospitals timely. Timeliness is an integral component in healthcare, along-with safety, effectiveness, and patient centeredness. ${ }^{5,6}$ Timeliness is defined as "the degree to which care is provided to the patient at the most beneficial or necessary time". ${ }^{7}$ Particularly in developing countries, timeliness related to maternal services was emphasized through the three-delay model, which identifies three types of delays that contribute to the increased probability of maternal mortality which are the delay in deciding to seek care, delay in reaching a health facility, and delay in receiving adequate treatment at the facility. ${ }^{8}$ While the first delay is mainly the decision of the family and subject to community norms, the other two can be mitigated by implementing telemedicine solutions. ${ }^{9,10}$

\section{Methods}

An online literature review was conducted for articles related to telemedicine, its use in maternal health services, and maternal mortality, where related Web pages were visited.

\section{Discussion}

The World Health Organization defines maternal death as the death of a pregnant woman due to complications related to pregnancy, underlying conditions worsened by the pregnancy or management of these conditions. This can occur either while she is pregnant or within six weeks of resolution of the pregnancy. ${ }^{11}$

Maternal mortality is highest in developing countries due to prevailing social norms that inform health seeking behavior, particularly that relate to reproductive and maternal health services, burdened health systems, and inadequate quality health care services, particularly in remote areas. ${ }^{12}$ These factors contribute to the three delays that increase the chance of maternal deaths. In the following 
section each delay will be described, and the suggested role of utilizing telemedicine solutions to mitigate these factors will be discussed.

The first delay; delay in decision to seek care, is caused by prevailing social norms that might in some cases rely on traditional and non-skilled attendants, coupled with limited understanding of pregnancy risk factors and danger signs that require medical assistance, and when to seek medical help. The financial inability to seek services, particularly if these service are not free of charge or if the services are far and require transportation is also a hindering factor in taking such decision.

The second delay; delay in reaching care, usually occurs when all the health service delivery points are far, or only the primary care health facilities are available, while the secondary and tertiary are far, the infrastructure of roads, and the geography of the place. ${ }^{13,14}$

The third delay; delay in receiving adequate health services, is mainly related to the adequacy and quality of the health system itself, as in poor, burdened health systems there are usually limited supplies, facilities, untrained and inadequate medical staff, coupled with poor referral mechanisms. ${ }^{13,14}$

In many cases these three delays can be combined together, for instance in many areas of the developing world there are scant health services, long distance to the central hospital, prevalence of social norms that might limit mobility of women, and on top of this comes poverty.

Through telemedicine solutions, most of these delays can be overcome, where connecting and assisting health care providers working in a facility in a remote are to specialised physicians in the nearest specialized facility, to receive guidance and advice on how to deal with childbirth complications and to respond to maternal health emergencies in the most efficient manner, or stabilize and effectively deal with the case till it reaches safely to the specialized facility.

It's imperative that the solution provides a comprehensive overview of the situation in real-time and respond efficiently to the emergency through a direct, user-friendly and easy connection established between the two sites by assisting in deliveries through sharing the most relevant data of the patients, the connected medical sensors, and the possibility of video calls and messages.

This model can be also implemented between the destination specialized hospital receiving the complicated childbirth cases and the ambulance driving the patient. Ambulance attendants usually have training on life-saving interventions, but might not be able to deal with cases like maternal hemorrhage. Connecting the ambulance with the specialist, and provision of directions to the ambulance attendant through live video conferencing is imperative to stabilize the case. Optimally, the ambulance attendant can be equipped with smart goggles which would enable a wider angle of examination, where the remote specialist will be able to better visualize the case.

Moreover, telemedicine consultations can overcome the delay in seeking services by having a trained on call service provider who can talk to the pregnant woman, or a family member, to provide guidance, and alert them if immediate care is required.

In view of the above, the main functionalities of the telemedicine solution are:

I. Video conference between health professionals in in lower and higher level facilities, or between the ambulance and the facility, or between the patient and the specialists.
II. Sensor's data such as ECG, CTG, and ultrasound, that can be shared with the specialists also during the video conference.

III. Patients Tab: For patient management and visualizations of patient data.

IV. Communication Tab: For messaging and communications regarding the patients.

V. Rota Management: For management of the rotation of doctors in the system.

VI. Mobile App for providers in the referral site to respond to and manage the emergencies.

Using the above features, in the primary health care level, if service providers receive a patient in emergency state, they can login to the telemedicine solution, connect the necessary vital sensors to the patient and enter the data and treatment for the patient in the platform. Then $\mathrm{s}(\mathrm{he})$ video calls the more advanced designated referral site, transmits patient data, and with the help of the camera streaming and the live sensor data, the specialized provider can assist.

At the referral site, or specialized hospital, the service providers receive a notification on the smartphone about the emergency, they can answer on the phone or send the call to the terminal unit of the telemedicine system, they can also login in the terminal unit, see the patient's situation and call back the ambulance or primary care unit to provide immediate assistance. Reading the sensor's data and discussing the diagnosis, concerned service providers - including the ambulance attendant - can agree and advise on the most necessary and appropriate treatment to address the emergency case.

\section{Limitations}

There are several limitations that would face effective implementation of the telemedicine solution in maternal and obstetric complications.

One of the major limitations is availability of equipment and infrastructure of all the three points of health care mentioned above, where the effective implementation of this solution requires screens, computers smart cameras, secured virtual private network (VPN) connection, secure electronic medical record application with virtual clinical solution to $\log$ in the cases and use the video conference through it for providing documentation and statistics reports, and smart goggles that supports Internet of Things (IOT) and embedded camera.

Another limitation would be political and technical support of policy makers such as health authorities, syndicates, etc., to implement, monitor and evaluate such solutions. Provision of training on the utilization of the required equipment might be a challenge. In burdened health systems, where wages are generally low, there might not be a motive for service providers to commit to the implementation of such solution.

\section{Conclusion}

Telemedicine solutions can be a significate life-saving intervention in complicated child birth situations that require emergency obstetric care. This solution can be able to mitigate the effects of the three delays that increase maternal deaths, as it goes beyond the usual patient to provider consultation, but provides a chance for untrained or non-specialized service providers to save the life of a woman and her baby using guided techniques, or at least stabilize the case, even in 
the ambulance till it reaches the higher level facility, who also became acquainted about the case and this would give the facility the chance to prepare in advance.

\section{Acknowledgments}

None.

\section{Conflict of interest}

The authors declare that they have no competing interests.

\section{Funding}

None.

\section{References}

1. Telemedicine: centers for Medicare and Medicaid services

2. Telemedicine: opportunities and developments in Member States: report on the second global survey on eHealth. 2009.

3. Heinzelmann PJ, Lugn NE, Kvedar JC. Telemedicine in the future. Journal of Telemedicine and Telecare. 2005;11(8):384-390.

4. Galle A, Semaan A, Huysmans E, et al. A double-edged sword-telemedicine for maternal care during COVID-19: findings from a global mixedmethods study of healthcare providers. BMJ Glob Health. 2021;6(2):4575.
5. Kerr EA, EA McGlynn, J Adams, et al. Profiling the quality of care in communities: Results from the community quality index study. Health Affairs. 2004; 23(3):247-256.

6. McGlynn EA, SM Asch, J Adams, et al. The quality of health care delivered to adults in the United States. New England Journal of Medicine. 2003;348(26):2635-2645.

7. Joint Commission on Accreditation of Healthcare Organizations. The Framework for Improving Performance: From Principles to Practice. Oakbrook, IL: Joint Commission. 1994.

8. Thaddeus S, Maine D. Too far to walk: maternal mortality in context. Soc Sci Med. 1994;38:1091-1110.

9. How telemedicine can help close the maternal health gap. 2020

10. Kimberly Fryer, Arlin Delgado, Tara Foti, et al. Implementation of Obstetric Telehealth During COVID-19 and Beyond, June 2020 Implementation of Obstetric Telehealth During COVID-19 and Beyond. Matern Child Health J. 2020;24(9):1104-1110.

11. Maternal deaths.

12. Harrison K. Maternal mortality in developing countries. 1989.

13. Maternity Worldwide. The Three Delays Model and our Integrated.

14. Shah N, Hossain N, Shoaib R, et al. Socio-demographic characteristics and the three delays of maternal mortality. $J$ Coll Physicians Surgeons Pak. 2009;19:95-98. 